\title{
Gestão de carteiras sob múltiplos regimes: Performance fora da amostra no mercado brasileiro
}

(Portfolio management under multiple regimes: Out-of-sample performance in the Brazilian market)

\author{
Marcelo Lewin ${ }^{\dagger}$ \\ Carlos Heitor Campani
}

\begin{abstract}
Resumo Propomos uma estratégia de alocação dinâmica para um investidor que considera três regimes econômicos latentes, estimados através dos retornos de cinco classes de ativos brasileiros. A estratégia é definida a partir de uma solução analítica aproximada numa configuração econômica realista. A performance da carteira supera o retorno de todos os benchmarks analisados em 6 dos 10 anos em tela, tendo apresentado média de retorno semanal superior a qualquer benchmark, com significância estatística. De 2010 a 2019, nossa estratégia alcança um retorno médio de 21,6\% ao ano contra, por exemplo, 9,8\% a.a. do CDI e 4,7\% a.a. do Ibovespa. A análise comparativa deixa claro, em especial, a importância de se considerar múltiplos regimes na estratégia de alocação.

Palavras-chave: Gestão de carteiras, Múltiplos regimes; Alocação dinâmica; Performance.

Código JEL: G11, G24, O16.

Abstract We propose a dynamic allocation strategy for an investor which considers three unobservable economic regimes, which we estimate using returns on five Brazilian asset classes. The strategy is based on an approximate analytical solution of a realistic configuration of the economy. The out-of-sample performance exceeds those of every benchmark we consider in 6 out of 10 years, with a weekly average return significantly higher than any benchmark at the usual confidence levels. From 2010 to 2019, our strategy achieves an average return of $21.6 \%$ per annum against, for example, $9.8 \%$ p.a. of the CDI and $4.7 \%$ p.a. of the Ibovespa. In particular, a comparative analysis makes clear how important it is to include multiple regimes in portfolio allocation.
\end{abstract}

Keywords: Portfolio management; Multiple regimes; Dynamic allocation; Performance. JEL Code: G11, G24, O16.

\section{Introdução}

Evidências empíricas sugerem que modelos econômicos que preveem mudanças de regimes (regime switching models) acrescentam relevante informa-

Submitted on March 20, 2020. Revised on May 30, 2020. Accepted on July 14, 2020. Published online in September 2020. Editor in charge: Yuri Saporito.

$\dagger$ Instituto COPPEAD de Administração - UFRJ, Brazil: marcelo. lewin@ coppead. ufrj.br

¥Instituto COPPEAD de Administração - UFRJ, Brazil and EDHEC - Risk Insitute:

carlos.heitor@coppead.ufrj.br 
ção à gestão de carteiras de investimento, estimando em tempo real as probabilidades de ocorrência de regimes em períodos vindouros que modelam os retornos, volatilidades e intercorrelações de ativos, como apontam Ang e Bekaert (2004), Tu (2010), Guidolin e Hyde (2012) e Ang e Timmermann (2012).

A revisão da literatura feita por Guidolin (2011) sugere que bastariam dois regimes para descrever a distribuição dos retornos de ações e intercorrelações; e apenas três regimes para descrever o mercado de renda fixa. De forma genérica, porém, a coerência entre regimes com diferentes classes de ativos e/ou regiões geográficas pode não ser perfeita, segundo Bae et al. (2014) e Case et al. (2014). Logo, uma economia com classes de investimento diversificadas pode se caracterizar por processos de mudanças de regimes não-perfeitamente sincronizados ou que apresentem outro número de regimes, como Guidolin e Timmermann (2007) que estimaram quatro regimes nos EUA a partir dos ativos: renda fixa de longo prazo, dois portfólios de ações (large e small caps) e a taxa livre de risco.

A contribuição desta pesquisa à literatura advém da estimação dos regimes a partir de um conjunto relevante e original de cinco classes de ativos com o viés do investidor brasileiro, e a avaliação de estratégia de alocação baseada no modelo de Campani et al. (2020) - doravante modelo CGL. Trabalhamos com as seguintes classes de ativos: cash (taxa livre de risco no Brasil), carrytrade (taxa livre de risco nos EUA convertida em reais), renda fixa de curto e longo prazo, e renda variável. O objetivo principal da pesquisa foi avaliar a performance do modelo CGL fora da amostra com um conjunto original de classes de ativos, estendendo os testes de robustez realizados por Lewin e Campani (2020) - estes com a limitação de terem sido dentro da amostra.

Como resultado primário, apesar de utilizarmos classes de investimento diversificadas como Guidolin e Timmermann (2007) e Lewin e Campani (2020), vemos que a influência das classes de renda fixa em nosso portfólio indica a estimação de apenas três regimes na aplicação do modelo, como sugere a literatura apresentada acima em referência ao mercado de renda fixa.

Como resultado principal, ao aplicarmos os parâmetros dos múltiplos regimes e o modelo de alocação CGL, tal estratégia apresenta um retorno médio semanal estatisticamente superior a todos benchmarks considerados nesta pesquisa no período analisado (2010 a 2019). Além disso, a carteira CGL logrou êxito ao obter o maior retorno dentre todos os benchmarks em 6 dos 10 anos analisados. Assim, concluímos que o modelo apresenta resultados empíricos que podem despertar o interesse de gestores de carteiras e de fundos de investimento no mercado. 


\section{Revisão da literatura}

A pesquisa seminal de Hamilton (1989) apresenta o modelo econométrico de mudanças de regimes onde a previsão dos estados econômicos (regimes) é realizada através de um sistema probabilístico de cadeias de Markov, originado a partir dos dados extraídos nos retornos dos ativos. Ang e Bekaert (2002) e Graflund e Nilsson (2003) foram as primeiras pesquisas a aplicar esta técnica na alocação de ativos. Ambas pesquisas identificaram dois regimes (bull e bear market), porém ignoraram a característica latente dos regimes, considerando-os totalmente observáveis. Posteriormente, Guidolin e Timmermann (2007) identificaram a existência de quatro regimes latentes e, utilizando a função potência de utilidade (power utility function), otimizaram carteiras de investimento através da simulação de Monte Carlo. Esta clássica função utilidade permite um tratamento matemático simplificado que poupa tempo na simulação, entretanto se distancia da realidade empírica ao prever uma relação injustificável entre os parâmetros de risco de alocação $(\gamma)$ e de consumo intertemporal $(\psi)$. Tal relação é apresentada por Munk (2013).

Campani et al. (2020) igualmente identificaram quatro regimes latentes aplicando o modelo de mudança de regimes sob a mesma configuração de ativos de Guidolin e Timmermann (2007). Porém, o modelo CGL considera, para efeitos de alocação de ativos, a função de utilidade estocástica diferencial de Duffie e Epstein (1992) - uma classe dentre as funções recursivas de utilidade, que devidamente separa os parâmetros de risco $\gamma$ e $\psi$.

Infelizmente, modelos realistas para seleção de carteiras ainda não contam com soluções analíticas exatas em economias com múltiplos regimes. Em consequência, a simulação de Monte Carlo (SMC) surge como opção natural: entretanto pode facilmente tornar-se inviável por conta do tempo de processamento. Isto abriu caminho para Campani et al. (2020) proporem uma solução analítica aproximada, baseada na aproximação proposta por Campani e Garcia (2019) em uma economia de único regime e função de utilidade estocástica diferencial: os autores demonstraram que a aproximação é suficientemente precisa para a alocação de ativos. Uma solução analítica permite a avaliação das estratégias de alocação de forma mais profunda - por exemplo: o impacto dos parâmetros de mercado pode ser verificado de maneira mais nítida - e superam o severo custo de tempo imposto pela SMC em configurações realistas.

O modelo CGL já foi aplicado no Brasil por Lewin e Campani (2020), que identificaram quatro regimes latentes na economia em quatro classes de ativos: renda fixa, renda variável nacional e renda variável internacional (convertida em reais), além da taxa livre de risco. Os autores mostram que os re- 
sultados da carteira CGL foram promissores, porém a análise foi feita dentro da amostra. A atual pesquisa inova ao avaliar a performance do modelo CGL fora da amostra, afinal "no trabalho aplicado, modelos dinâmicos de séries temporais serão apenas tão bons quanto sua performance preditiva", ou seja, fora da amostra (Guidolin, 2011).

Os regimes e a carteira são estimados a partir de cinco classes de ativos: taxa livre de risco, carry-trade sob a ótica de um investidor brasileiro (taxa livre de risco dos EUA convertida em reais), renda fixa de longo e de curto prazo, e renda variável. A estratégia de alocação se mostrou um pouco mais complexa pelo maior número de ativos em relação a Lewin e Campani (2020), e devido ao aumento da participação das classes de renda fixa na carteira.

Sob esta configuração, identificamos três regimes latentes e propomos a estratégia do modelo CGL com vendas a descoberto e horizonte finito. Mostraremos que os resultados são relevantes em relação aos principais benchmarks brasileiros. Com a estimação fora da amostra, os resultados podem também ser obtidos por gestores de ativos e a ambição deste trabalho é exatamente oferecer a aplicação de um modelo pioneiro para gerir carteiras no mercado brasileiro de forma mais eficaz, sob a ótica de um modelo de múltiplos regimes econômicos.

\section{Metodologia e dados}

\subsection{Prêmios de risco}

Acompanhando os desenvolvimentos econométricos descritos em Campani et al. (2020) e Lewin e Campani (2020), configuramos um modelo de tempo contínuo, discretizado para efeito prático em semanas - o que será justificado adiante. Consideramos um mercado eficiente e sem saltos, incorporando $n+1$ classes de ativos: um ativo de curto prazo livre de risco e $n$ ativos de risco. Utilizamos prêmios de risco para absorver a volatilidade do ativo livre de risco nos retornos dos ativos de risco. Calculamos os retornos em excesso $\left(\widehat{r}_{i, t+1}\right)$, também denotados como prêmio de risco, de $t$ para $t+1$, com $t$ medido em semanas:

$$
\hat{r}_{i, t+1}=\frac{1+r_{i, t+1}}{1+r f_{t+1}}-1,
$$

com

$$
r_{i, t+1}=\frac{s_{i, t+1}-s_{i, t}}{s_{i, t}}
$$

onde $i=1,2, \ldots, n ; S_{i, t}$ é preço do ativo de risco $i$ na semana $t$, o qual incorpora dividendos e quaisquer outros efeitos de retorno; $r_{i, t+1}$ é o retorno total 
do ativo de risco $i ; r f_{t+1}$ é o retorno do ativo livre de risco na semana de $t$ a $t+1$.

\subsection{Configuração da economia e estimação dos parâmetros}

Consideramos que a dinâmica dos prêmios de risco dos $n$ ativos pode ser definida pelo seguinte processo estocástico multidimensional:

$$
\left[\begin{array}{c}
\mathrm{d} \hat{r}_{1, t} \\
\mathrm{~d} \hat{r}_{2, t} \\
\ldots \\
\mathrm{d} \hat{r}_{n, t}
\end{array}\right]=\left[\begin{array}{c}
\mu_{1, t} \\
\mu_{2, t} \\
\ldots \\
\mu_{n, t}
\end{array}\right] \mathrm{d} t+\left[\begin{array}{cccc}
\sigma_{11, t} & 0 & \ldots & 0 \\
\sigma_{21, t} & \sigma_{21, t} & \ldots & 0 \\
\ldots & \ldots & \ldots & \ldots \\
\sigma_{n 1, t} & \sigma_{n 2, t} & \ldots & \sigma_{n n, t}
\end{array}\right]\left[\begin{array}{c}
\mathrm{d} Z_{1, t} \\
\mathrm{~d} Z_{2, t} \\
\ldots \\
\mathrm{d} Z_{n, t}
\end{array}\right],
$$

com as seguintes definições convenientes:

$$
\boldsymbol{\mu}_{s, t}=\left[\begin{array}{c}
\mu_{1, t} \\
\mu_{2, t} \\
\ldots \\
\mu_{n, t}
\end{array}\right] ; \boldsymbol{\sigma}_{s, t}=\left[\begin{array}{cccc}
\sigma_{11, t} & 0 & \ldots & 0 \\
\sigma_{21, t} & \sigma_{21, t} & \ldots & 0 \\
\ldots & \ldots & \ldots & \ldots \\
\sigma_{n 1, t} & \sigma_{n 2, t} & \ldots & \sigma_{n n, t}
\end{array}\right] ; \mathrm{d} \boldsymbol{Z}_{t}=\left[\begin{array}{c}
\mathrm{d} Z_{1, t} \\
\mathrm{~d} Z_{2, t} \\
\ldots \\
\mathrm{d} Z_{n, t}
\end{array}\right],
$$

onde $\boldsymbol{\mu}_{s, t}$ corresponde ao vetor coluna $(n \times 1)$ contendo os prêmios de risco instantâneos esperados (drifts); $\boldsymbol{\sigma}_{s, t}$ corresponde à matriz de volatilidades $(n \times n)$ desenhada como uma matriz triangular inferior (sem absolutamente nenhuma perda de generalidade) e $\mathrm{d} \boldsymbol{Z}_{t}$ corresponde ao vetor coluna $(n \times 1)$ contendo $n$ incrementos de processos de Wiener padronizados e independentes entre si.

$\mathrm{O}$ vetor dos prêmios de risco $\left(\boldsymbol{\mu}_{s, t}\right)$ e a matriz de volatilidades $\left(\boldsymbol{\sigma}_{s, t}\right)$ variam no tempo e em função da variável de estado $Y_{t}$ (não-observável). Definimos que a variável de estado $Y_{t}$ refere-se à transição entre os regimes como um processo de cadeias de Markov independente, em tempo contínuo, que é contínua para o limite da direita e admite apenas valores em $R=\{1,2, \ldots, m\}$ onde $R$ corresponde ao conjunto finito de $m$ possíveis regimes econômicos. No estado $Y_{t}=i, i \in R$, temos:

$$
\boldsymbol{\mu}_{s, t}=\left[\begin{array}{c}
a_{1, i} \\
a_{2, i} \\
\ldots \\
a_{n, i}
\end{array}\right] \quad \text { e } \quad \boldsymbol{\sigma}_{s, t}=\boldsymbol{\sigma}_{s, i}=\left[\begin{array}{cccc}
\sigma_{11, i} & 0 & \ldots & 0 \\
\sigma_{21, i} & \sigma_{21, i} & \ldots & 0 \\
\ldots & \ldots & \ldots & \ldots \\
\sigma_{n 1, i} & \sigma_{n 2, i} & \ldots & \sigma_{n n, i}
\end{array}\right],
$$

com coeficientes $a_{j, i}$ e matrizes $\boldsymbol{\sigma}_{s, i}$ constantes para cada $i \in R$ e $j=1,2, \ldots, n$. Ressaltamos que $\boldsymbol{\sigma}_{s, i} \boldsymbol{\sigma}_{s, i}^{T}$ representa a matriz de variância-covariância dos retornos dos ativos sob o regime $i$. Todos os elementos de $\boldsymbol{\sigma}_{s, i}$ são definidos 
como volatilidades parciais: por exemplo, $\sigma_{21, i}$ representa a volatilidade parcial do ativo 2 com relação ao primeiro processo de Wiener $\left(\mathrm{d} Z_{1, t}\right)$ no regime $i$.

Os drifts dos prêmios de risco dependem dos regimes e simultaneamente variam no tempo, mesmo que o regime permaneça inalterado. Eles são coletados na matriz de drifts (que varia no tempo) $\boldsymbol{D}_{s, t}$ cujas dimensões são $n \times m$ :

$$
\boldsymbol{D}_{s, t}=\left[\begin{array}{cccc}
a_{1,1} & a_{1,2} & \ldots & a_{1, m} \\
a_{2,1} & a_{2,2} & \ldots & a_{2, m} \\
\ldots & \ldots & \ldots & \ldots \\
a_{n, 1} & a_{n, 2} & \ldots & a_{n, m}
\end{array}\right] .
$$

Voltando à configuração da variável de estado $Y_{t}$, ela governa os estados da economia e não é observável. O processo pode começar em qualquer momento $t_{0}$ sob qualquer regime, que $Y_{t}$ permanecerá no mesmo regime por um período de tempo exponencialmente distribuído até saltar para outro regime. A permanência e os saltos entre regimes são tratados através de probabilidades de transição. Considerando que o regime vigente é $i$, a probabilidade de saltar para outro regime $j$ no período de tempo $\Delta t$ é dada por

$$
P_{i j, \Delta t}=\frac{\lambda_{i j}}{\sum_{k \neq i} \lambda_{i k}}\left(1-e^{-\sum_{k \neq i} \lambda_{i k} \Delta t}\right),
$$

onde $j \neq i \in R$ e $\lambda_{i j}>0$. Definimos $\lambda_{i i}=-\sum_{k \neq i} \lambda_{i k} \leq 0$ de forma que $P_{i j, \Delta t}=$ $\lambda_{i j}\left(1-e^{\lambda_{i i} \Delta t} /\left(-\lambda_{i i}\right)\right)$. Assim, a probabilidade de permanecer no mesmo regime $i$ no próximo período de tempo $\Delta t$ será $P_{i i, \Delta t}=e^{\lambda_{i i} \Delta t}$. Sob a premissa de que sejam constantes, os parâmetros $\lambda_{i j}$ representam a densidade da probabilidade de transição entre regimes.

Com base nestes processos, estimamos os parâmetros do modelo de regimes pelo método da máxima verossimilhança (MLE - Maximum Likelihood Estimation) de acordo com o Hamilton (1989), onde os parâmetros estimados são: os elementos da matriz $\boldsymbol{D}_{s, t}$ referente aos drifts dos ativos por regime; os elementos da matriz de volatilidades por regime $\boldsymbol{\sigma}_{s, i}$; e as probabilidades de transição entre os regimes $P_{i j, \Delta t}$ (onde $j \neq i \in R$ ). Na próxima seção, apresentaremos os valores de cada parâmetro estimado. Aqui ressaltamos que o total de parâmetros desta aplicação pode ser calculado como: $m \times n+m \times(n+1) \times n \times 0,5+m \times(m-1)$. Logo, sob $m=3$ regimes e $n=4$ ativos de risco, esta configuração demandou a estimação de 48 parâmetros.

Guidolin e Ono (2006) apontam que um modelo parcimonioso em termos de número de observações e regimes apresenta um índice de saturação superior à 26. Esta medida representa a quantidade de observações disponíveis por 
parâmetro estimado e é obtida através do número de ativos $(n)$ multiplicado pela quantidade de observações dividido pelo total de parâmetros.

Como nossa pesquisa objetivava a realização de backtests para calcular a performance com estimações fora da amostra, um número alto de regimes poderia inviabilizar o projeto pois a série restritiva se estendia apenas até set/2003. Para encontrar o número ótimo de regimes e definir o período de análise realizamos 563 otimizações (MLE) acompanhando a restrição imposta pelo índice de saturação. Testamos modelos com $m=3,4,5,6$ regimes sob diversas janelas de observação, com dados mensais, semanais e diários. Avaliamos todos os resultados através dos parâmetros de output da otimização, como: $f$-value, exit flag e número de iterações.

A estimação mais robusta consistentemente ocorria sob $m=3$ regimes e dados semanais. Com o índice de saturação superior às demais configurações, nesta, os parâmetros estimados não se alteravam significativamente nos testes de stress aos quais submetemos o modelo ao variar as janelas de dados. Em contrapartida, a série com dados mensais encurtava sobremaneira o número de observações, e a série diária não agregava melhora nos indicadores da estimação.

Ao rodarmos estimações com $m=4,5$ e 6 , encontramos sistematicamente 3 regimes "ativos" (com características similares ao modelo $m=3$ ) mas de acordo com as probabilidades filtradas mais regimes praticamente não ocorriam. As probabilidades filtradas, sendo aquelas que indicam o regime de $t+1$ em $t$, mostravam valores significativos apenas para 3 regimes ao passo que nos demais regimes tais valores tendiam à zero. Na seção a seguir discutiremos características especificas da carteira que favoreceram à estimação de "apenas" 3 regimes.

Não obstante, o índice de saturação também foi utilizado para estabelecermos o início das janelas como a primeira data disponível (set/2003) e aumentarmos gradativamente o total de observações. Desse modo, começamos com um índice de saturação de 27 na primeira janela (até dez/2009) e alcançarmos o índice de saturação de 71 na última janela (até dez/2019).

\subsection{Configuração do modelo de alocação}

Para realizar a estratégia de alocação, configuramos um investidor que maximiza o valor esperado da utilidade de seus investimentos em cada momento $t$ através da função recursiva de utilidade estocástica diferencial de Duffie e Epstein (1992) - que se destaca da função Epstein-Zin por operar no 
campo de contínuo (Munk, 2013):

$$
J_{t}=E_{t}\left[\int_{u=t}^{T} f\left(C_{u}, J_{u}\right) \mathrm{d} u+\frac{W_{T}^{1-\gamma}}{1-\gamma}\right],
$$

onde $E_{t}$ corresponde ao valor esperado condicional à data $t ; T$ é o horizonte do investimento e $t$, o instante atual; a função $f$ é o agregador de recursividade da função utilidade $\left(J_{t}\right)$, dado em função de $C_{u}$ que denota a taxa de consumo no momento $u$ que satisfaz o investidor, e $J_{u}$ representando a utilidade continuada em $u$; por sua vez, $W_{T}$ representa a riqueza do investidor no horizonte de investimento $T$ e $\gamma$, seu parâmetro de aversão a risco. Na equação (6) abaixo, detalha-se o agregador da função utilidade (descrito de forma genérica em função de duas variáveis $C$ e $J$ ):

$$
f(C, J)=\frac{\beta}{1-\frac{1}{\psi}}(1-\gamma) J\left\{\left[\frac{C}{[(1-\gamma) J]^{\frac{1}{1-\gamma}}}\right]^{1-\frac{1}{\psi}}-1\right\}
$$

onde $\beta$ é a taxa de preferência no tempo; e $\psi$ é a elasticidade intertemporal de substituição, i.e., parâmetro de risco de consumo intertemporal. Considera$\operatorname{mos} \psi=\infty$ a fim de eliminar o consumo e restringir o problema da pesquisa à alocação dos ativos. Seguindo Campani et al. (2020) e Guidolin e Timmermann (2007), consideramos $\gamma=5$ e $\beta=2 \%$ ao ano. Na fórmula acima, $C$ representa a taxa de consumo instantânea e $J$, a função utilidade (note-se a recursividade da função de utilidade, pois esta depende de si mesma nos momentos futuros).

Assumindo regimes latentes, ou seja, não observáveis, os investidores estimam probabilidades de ocorrência de cada regime no período seguinte, para o qual precisam tomar a decisão de alocação, em um vetor unidimensional $\left(\boldsymbol{\pi}_{t}\right)$. Como demonstram Campani et al. (2020), a solução geral que quantifica a utilidade total otimizada $\left(V_{t}=\sup J_{t}\right)$ do investidor a cada momento $(t)$ admite a solução separável da riqueza $\left(W_{t}\right)$ no seguinte formato:

$$
V\left(W_{t}, \boldsymbol{\pi}_{t}, \tau\right)=H\left(\boldsymbol{\pi}_{t}, \tau\right) \frac{W_{t}^{1-\gamma}}{1-\gamma}
$$

onde $\tau=T-t$ é o tempo restante até o horizonte final de tempo e $H\left(\boldsymbol{\pi}_{t}, \tau\right)$ é função deste mesmo horizonte e do vetor de probabilidades dos regimes. Uma expressão analítica exata para $H\left(\boldsymbol{\pi}_{t}, \tau\right)$ ainda não foi encontrada na literatura e, portanto, Campani et al. (2020) propõem uma expressão analítica 
aproximada para esta função. Eles mostram que tal aproximação é suficientemente precisa para alcançar a alocação ótima de portfólio com a maximização da utilidade. A função $H$ pode então ser aproximada da seguinte forma:

$$
H\left(\boldsymbol{\pi}_{t}, \tau\right)=\exp \left[A_{0}(\tau)+\sum_{i=1}^{m} A_{i}(\tau) \pi_{i, t}+\sum_{i=1}^{m} B_{i}(\tau) \pi_{i, t}^{2}+\sum_{j \neq i} C_{i j}(\tau) \pi_{i, t} \pi_{j, t}\right] .
$$

Com a solução aproximada para $V\left(W_{t}, \boldsymbol{\pi}_{t}, \tau\right)$, é possível resolver a equação de Bellman equivalente ao problema de otimização em tela para se obter os coeficientes $A_{0}, A_{i}, B_{i}$ e $C_{i j}$ da função $H\left(\boldsymbol{\pi}_{t}, \tau\right)$, todos eles dependentes do horizonte $\tau$ de investimento. $\mathrm{O}$ documento suplementar a este artigo $^{1}$ mostra o passo a passo matemático para a solução desta equação.

Desse modo, a carteira de investimento é otimizada e os pesos ótimos $\left(\boldsymbol{\alpha}_{t}\right)$ do modelo de múltiplos regimes em cada momento $t$ são definidos pela equação (9):

$$
\begin{aligned}
\boldsymbol{\alpha}_{t}= & \frac{1}{\gamma}\left(\boldsymbol{D}_{s, t} \boldsymbol{\pi}_{t}\right)^{T}\left[\left(\boldsymbol{V} \boldsymbol{\pi}_{t}\right)\left(\boldsymbol{V} \boldsymbol{\pi}_{t}\right)^{T}\right]^{-1} \\
& +\frac{1}{\gamma} \sum_{i=1}^{m}\left[A_{i}(\tau)+2 B_{i}(\tau) \pi_{i, t}+\sum_{j \neq i} C_{i j}(\tau) \pi_{i, t}\right] \boldsymbol{\sigma}_{i, \boldsymbol{\pi}}\left(\boldsymbol{V} \boldsymbol{\pi}_{t}\right)^{-1},
\end{aligned}
$$

onde $\boldsymbol{D}_{s, t}$ é a matriz $n \times m$ que coleta os prêmios de risco de cada ativo de risco em suas linhas, com as colunas indicando tais prêmios de risco em cada um dos $m$ regimes; $\pi_{i, t}$ é a probabilidade de se estar sob efeito do regime $i$ no período $t ; \boldsymbol{\pi}_{t}$ é um vetor coluna $m \times 1$ que contém as $m$ probabilidades de se estar, no período $t$, sob efeito de cada um dos $m$ regimes; $\boldsymbol{V}$ é um vetor $1 \times m$ que contém como seus elementos $m$ matrizes de covariância dos ativos condicionadas a cada um dos $m$ regimes; $A_{i}, B_{i}$ e $C_{i j}$ são coeficientes dependentes do horizonte temporal que advêm da solução de otimização do sistema de equações diferenciais parciais oriundos da equação de Bellman; e, finalmente, $\boldsymbol{\sigma}_{i, \boldsymbol{\pi}}$ é um vetor linha $1 \times n$ que coleta, para cada regime $i=1,2, \ldots m$, as volatilidades parciais $\sigma_{i j, \pi} \operatorname{com} j=1,2, \ldots n ; \boldsymbol{\sigma}_{i, \pi}=\left[\sigma_{i 1, \pi} \sigma_{i 2, \pi} \ldots \sigma_{i n, \pi}\right]$. Os apêndices A.1 e A.2 fornecem mais detalhes matemáticos da configuração da economia bem como do processo de estimação dos parâmetros do modelo.

\subsection{Alocação fora da amostra e rebalanceamento da carteira}

Consideramos que nosso investidor aloca seus recursos baseado na informação corrente e aguarda até o período seguinte para refazer suas escolhas

${ }^{1}$ Disponível em http://bibliotecadigital.fgv.br/ojs/index.php/rbfin/ 
quando receberá novas informações do mercado, ou seja, quando conhecer os retornos daquele período. Cabe um comentário muito importante aqui: ao contrário de Lewin e Campani (2020), onde os autores estimam o modelo uma única vez com dados de toda a amostra, neste estudo reestimamos o modelo semestralmente e apenas com as informações disponíveis naquele momento.

Para definir a estratégia, i.e., calcular os pesos na carteira de investimento, estimamos o modelo de 2009 até 2019, com a janela de informações fixada no início do período de análise e crescendo com o avançar do tempo. Esta janela temporal para estimação fora da amostra corresponde a utilização de cerca de $40 \%$ dos dados para calibragem (2001 até 2008) e 60\% dos dados para gerar os resultados. O processo de atualização do modelo, isto é, a nova estimação dos parâmetros dos regimes, foi sempre realizado no primeiro fechamento semanal dos meses junho e dezembro. Por exemplo, ao estimarmos os parâmetros do modelo após o primeiro fechamento em dezembro de 2009, mantemos esses parâmetros até a última semana de maio de 2010, quando então repetimos o procedimento para o semestre seguinte com uma nova estimação realizada após o fechamento da primeira quarta-feira de junho de 2009. Isto torna os resultados aqui encontrados fora da amostra, ou seja, totalmente replicáveis por investidores no dia a dia - este é mais um ingrediente pioneiro deste artigo.

A respeito do período de rebalanceamento da carteira de investimento, quanto menor for este período, mais preciso ele será em relação ao modelo contínuo para o qual a solução analítica foi originalmente encontrada, porém maiores os custos de transação e de implementação da estratégia. Tais custos não foram incluídos, pois dependem (e muito) das características do investidor. Por consistência, nossos benchmarks igualmente não terão tais custos considerados.

\subsection{Classes de ativos}

Utilizamos a taxa do Certificado de Depósito Interfinanceiro (CDI) para denotar o investimento livre de risco (cash), pois além de representar um investimento amplamente difundido no Brasil, muito comumente é assim considerada em detrimento da taxa Selic, como apontam Andrino e Leal (2018). Não obstante, os resultados aqui encontrados não são sensíveis à mudança para a taxa Selic. Isto não causa espanto tendo em vista que ambas as taxas apresentaram comportamentos extremamente similares no período aqui analisado.

Os quatro ativos de risco foram representados por índices (ou taxas). Os dados foram extraídos do terminal Economática $\odot$, exceto a taxa livre de risco estadunidense, extraída do sistema Board of Governors of the Federal Re- 
serve. Utilizamos fechamentos às quartas-feiras, compreendendo 849 observações de 24 de setembro de 2001 até 25 de dezembro de 2019. Nas datas em que não houve cotações, consideramos o fechamento anterior mais próximo. Optamos pela quarta-feira, ao invés da tradicional sexta-feira, para minimizar o efeito final de semana.

O Ibovespa foi adotado como a renda variável por ser um índice da B3 amplamente utilizado para representar o mercado brasileiro de ações, segundo Oliveira e Pereira (2018). Como investimento alternativo negativamente correlacionado à renda variável, denotamos como carry-trade a taxa do US Treasury Bill de 3 meses (T-Bill) convertido em reais pela taxa PTAX, computada pelo Banco Central do Brasil. Por último, segmentamos a classe de renda fixa entre IMA-B 5 (curto prazo) e IMA-B 5+ (longo prazo). Estes dois índices são calculados pela Anbima e compostos por títulos do Governo brasileiro (NTN-B) pós-fixados lastreados pelo índice inflacionário IPCA. Ressalte-se que o IMA-B 5, por possuir títulos com até 5 anos de vencimento, apresenta retorno e volatilidade muito semelhantes e alta correlação com o IRF-M (também calculado pela Anbima), que por sua vez, representa uma carteira com títulos pré-fixados (LTN e NTN-F). Por conta disso, o IRF-M foi desconsiderado como classe de ativos: ao incluí-lo, o modelo não era estimado de forma tão robusta e oscilações injustificáveis ocorriam por conta da alta correlação com o IMA-B 5.

Reconhecemos certo sobrepeso das classes de renda fixa em relação à renda variável na carteira selecionada: de um lado há dois ativos referentes a títulos do governo brasileiro e o CDI, e do outro, um ativo referente às ações brasileiras. Entretanto, há de se ponderar em separado a classificação atribuída ao carry-trade convertido em reais. Ao formar esta classe, verificamos que a PTAX apresenta impacto muito mais significativo do que o retorno da T-Bill. O objetivo de criá-la foi representar a alternativa do investidor brasileiro, que compra dólares para se proteger - e quando o faz, indiretamente aplica em algum investimento. Selecionamos a taxa livre de risco dos EUA para resumir o risco da classe apenas ao câmbio. Apesar da PTAX/T-Bill não ser formalmente uma renda variável como ações, a classe encontra forte correlação negativa com o IBOV. Portanto, em relação à composição da carteira, poderíamos separar 3 ativos ligados à renda fixa e 2 ativos ligados à renda variável.

Não obstante, os aspectos que motivaram tal seleção de ativos foram: a importância do mercado de renda fixa brasileiro e a pesquisa realizada para se chegar nesta carteira. Com o objetivo de realizar backtests fora da amostra, buscamos uma carteira comum a todos os anos da amostra, sem alterar os ativos a cada ano. O mercado de renda fixa nacional, embora a taxa básica de 
juros tenha sofrido cortes desde meados de 2016-17, possui relevante importância frente à renda variável no Brasil. Como proxy, tomemos os dados sobre a indústria de fundos de investimento no Brasil (Anbima, 2020). O histórico mostra que o patrimônio líquido dos fundos de renda fixa deteve participação significativamente maior do que as demais classes de investimentos em todos os anos entre dez/2009 e dez/2019. Por fim, o outro aspecto determinante à seleção das classes de ativos adveio da pesquisa realizada em 2019 junto a fundos de pensão, que alertaram possuir (até aquele momento) restrições quanto à exposição a ativos de renda variável no exterior e a ativos de crédito privado.

\subsection{Abordagens utilizadas na avaliação da performance}

Para avaliar a performance da carteira sugerida pelo modelo de múltiplos regimes (modelo CGL), observamos os resultados dos índices representativos das classes de ativos (CDI, IBOV, PTAX+T-Bill, IMA-B 5+ e IMA-B 5); e criamos duas carteiras de referência. A primeira utiliza a função estocástica diferencial, mas enxerga apenas um regime na economia (regime único); e a segunda representa a carteira igualmente ponderada, ou seja, sempre rebalanceada uniformemente para $20 \%$ em cada classe (denotada como $1 / n$ ou pesos iguais).

A comparação do modelo CGL com a carteira de regime único, também classificada como carteira míope por não enxergar os múltiplos regimes, é relevante na medida em que ambos os modelos utilizam a mesma função utilidade. Portanto, esta comparação permite avaliar a importância do modelo de múltiplos regimes na alocação de ativos.

Já a carteira igualmente ponderada denota uma média linear dos retornos dos ativos, o que, em última instância, representa um investidor que não possui um modelo de alocação superior à própria média. DeMiguel et al. (2009) recomendam a utilização desta carteira como benchmark para a performance de portfólios ao invés de outros modelos que otimizam a alocação para eliminar da comparação a possibilidade de erros de estimação. Na mesma linha, Kessler e Scherer (2009) afirmam que uma carteira de pesos iguais permite avaliar de forma mais clara as diferenças entre performances comparadas ao invés de um portfólio de média-variância, por exemplo, uma vez que este demandaria a estimação de uma matriz de covariâncias e eventuais erros de estimação prejudicariam a comparação com outros métodos. No Brasil, Santiago e Leal (2015) mostram a relevância da carteira de pesos iguais através de sua comparação empírica com 52 fundos de ações: apenas 2 fundos superaram os retornos da carteira $1 / n$. Não obstante, Andrino e Leal (2018) sugerem que portfólios de renda fixa com pesos iguais podem superar fundos 
balanceados. Desse modo, a literatura sugere a comparação com a carteira de pesos iguais para se avaliar a importância de um modelo na gestão ativa de portfólios. No caso, a gestão ativa se refere às estratégias de portfólio que visam maximizar retornos permitindo rebalanceamentos dinâmicos na alocação dos ativos da carteira.

\title{
4. Resultados, análises e discussões
}

\subsection{Modelo de múltiplos regimes}

A Tabela 1 apresenta os parâmetros dos regimes identificados na última estimação do modelo, em 04.12.2019. Observamos que o regime 1 é o mais volátil e apesar de geralmente isto indicar um movimento pessimista da economia, eventualmente compreende situações de forte volatilidade positiva, o que é comumente conhecido no mercado como um "rally". O regime 2 engloba a maioria das flutuações do mercado, enquanto o regime 3 denota a alta da renda variável junto com o CDI rendendo acima das classes de juros.

\section{Figura 1}

Probabilidades históricas de ocorrência dos regimes
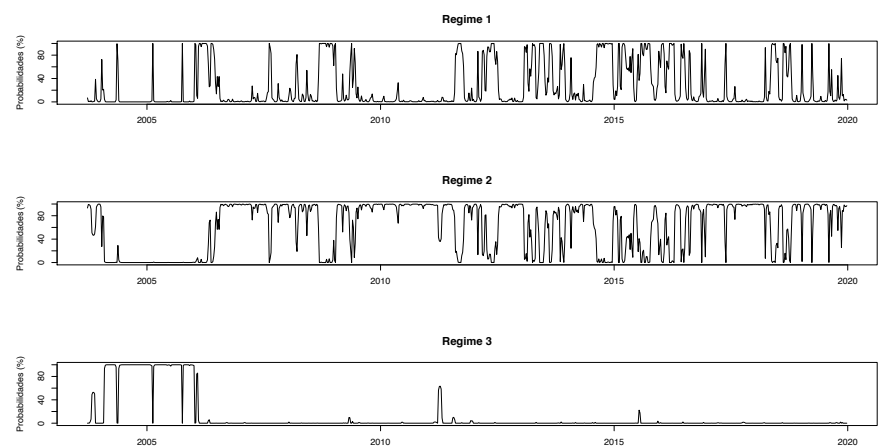

\begin{abstract}
A figura ilustra as probabilidades históricas semanais de ocorrência de cada regime. Pelo caráter informacional (e não de investimento), trata-se da probabilidade suavizada, isto é, que reúne todas as informações do período completo até dezembro de 2019. Os dados correspondem à última estimação do modelo, realizada em 04.12.2019.
\end{abstract}

A Figura 1 ilustra a probabilidade histórica dos três regimes identificados. Fica nítido que o terceiro regime mostra-se "adormecido" na maior parte do tempo. Isto ocorre pois o modelo atribui ao terceiro regime o estado da economia observado em meados de 2005, quando o Brasil vivia um super ciclo nas commodities, de forma que posteriormente tal regime ficou subutilizado, operando o modelo mormente com apenas dois regimes. 


\section{Tabela 1}

Parâmetros estimados para os modelos de múltiplos regimes em 4 de dezembro de 2019

IBOV PTAX+T-Bill IMA-B 5+

IMA-B 5

\begin{tabular}{|c|c|c|c|c|}
\hline \multicolumn{5}{|c|}{ Painel A: modelo míope (enxerga um único regime na economia) } \\
\hline $\begin{array}{l}\text { retorno médio dos prêmios de risco } \\
\text { matriz de correlação e volatilidade }\end{array}$ & $4 \%$ & $-8 \%$ & $6 \%$ & $2 \%$ \\
\hline IBOV & $24 \%$ & & & \\
\hline PTAX+T-Bill & $-50 \%$ & $15 \%$ & & \\
\hline IMA-B 5+ & $36 \%$ & $-41 \%$ & $10 \%$ & \\
\hline IMA-B 5 & $29 \%$ & $-31 \%$ & $77 \%$ & $3 \%$ \\
\hline \multicolumn{5}{|l|}{ Painel B: modelo de múltiplos regimes } \\
\hline \multicolumn{5}{|l|}{ retorno médio dos prêmios de risco } \\
\hline regime 1 & $-33 \%$ & $36 \%$ & $-6 \%$ & $-1 \%$ \\
\hline regime 2 & $21 \%$ & $-17 \%$ & $12 \%$ & $4 \%$ \\
\hline regime 3 & $16 \%$ & $-28 \%$ & $-2 \%$ & $-2 \%$ \\
\hline \multicolumn{5}{|l|}{$\begin{array}{l}\text { matriz de correlação e volatilidade } \\
\text { regime } 1\end{array}$} \\
\hline IBOV & $33 \%$ & & & \\
\hline PTAX+T-Bill & $-54 \%$ & $24 \%$ & & \\
\hline IMA-B 5+ & $45 \%$ & $-48 \%$ & $16 \%$ & \\
\hline IMA-B 5 & $39 \%$ & $-37 \%$ & $78 \%$ & $4 \%$ \\
\hline \multicolumn{5}{|l|}{ regime 2} \\
\hline IBOV & $20 \%$ & & & \\
\hline PTAX+T-Bill & $-47 \%$ & $10 \%$ & & \\
\hline IMA-B 5+ & $25 \%$ & $-28 \%$ & $6 \%$ & \\
\hline IMA-B 5 & $19 \%$ & $-20 \%$ & $75 \%$ & $2 \%$ \\
\hline \multicolumn{5}{|l|}{ regime 3} \\
\hline IBOV & $22 \%$ & & & \\
\hline PTAX+T-Bill & $-30 \%$ & $11 \%$ & & \\
\hline IMA-B 5+ & $-7 \%$ & $6 \%$ & $1 \%$ & \\
\hline IMA-B 5 & $6 \%$ & $-8 \%$ & $52 \%$ & $1 \%$ \\
\hline probabilidades de transição semanal & regime 1 & regime 2 & regime 3 & duração média \\
\hline regime 1 & $75 \%$ & $23 \%$ & $2 \%$ & 4 semanas \\
\hline regime 2 & $9 \%$ & $90 \%$ & $1 \%$ & 10 semanas \\
\hline regime 3 & $6 \%$ & $1 \%$ & $93 \%$ & 15 semanas \\
\hline
\end{tabular}

Nota: A tabela mostra as classes de ativos de risco. Os retornos estão configurados como prêmios de risco em relação ao CDI (classe cash). As matrizes de correlação apresentam as volatilidades em suas diagonais principais. Retornos e volatilidades originam-se dos dados semanais e foram anualizados para apresentação. 


\subsection{Modelo de alocação}

A Figura 2 apresenta um aspecto relevante da estratégia de alocação que é a forte alavancagem indicada pelo modelo CGL. A literatura aponta que modelos similares também apresentam esse resultado em função do maior conhecimento em relação aos estados econômicos (Sangvinatsos e Wachter, 2005). Isto faz com que o investidor tome posições bastante alavancadas. Não obstante, Lewin e Campani (2020) mostram que um menor nível de alavancagem não altera a formatação da carteira CGL, porém apenas a magnitude de seus retornos. Desse modo, utilizamos um maior nível alavancagem para apresentar o potencial do modelo. Consideramos que as posições vendidas em títulos de renda fixa ou cash, por exemplo, podem ser replicadas com posições equivalentes no mercado brasileiro de derivativos de juros.

Figura 2

\section{Distribuição dos pesos na carteira CGL}

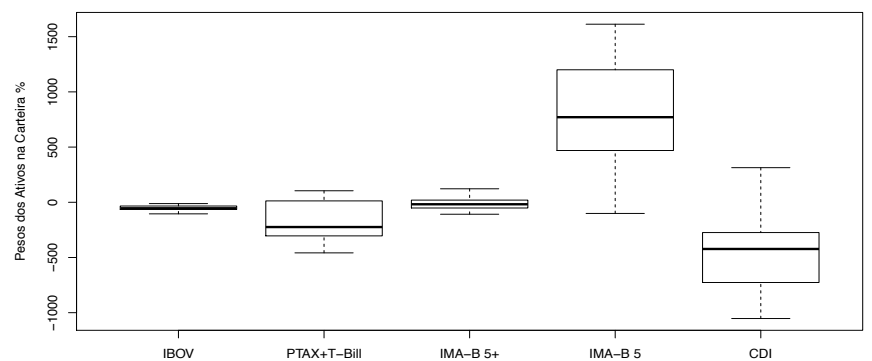

A figura ilustra a distribuição dos pesos na carteira formada pelo modelo CGL com base na estimação fora da amostra durante a janela de observações de 02.12.2009 até 04.12.2019. Outliers foram suprimidos da imagem.

A Figura 2 mostra que o modelo frequentemente indica forte posição comprada na classe de juros de curto prazo (IMA-B 5), que por sua vez possui alta correlação com juros prefixados. Em contrapartida, as maiores posições vendidas a descoberto são no ativo livre de risco (CDI) e depois no ativo de juros estadunidenses convertido em reais (PTAX+T-Bill): isso indica que o maior conhecimento a respeito dos regimes faz com que o investidor tome emprestado no Brasil e nos EUA para alavancar posições nos mercados de renda fixa e variável brasileiros.

Por outro lado, o modelo opera com volume nitidamente menor tanto na renda variável (IBOV) quanto nos juros de longo prazo (IMA-B 5+). No caso da renda variável, a maior proporção de renda fixa na carteira "força" o modelo na direção de três regimes. Isto é encontrado na literatura em Guidolin 
(2011) que aponta que a estimação de "apenas" três regimes implica em menor coerência na diversificação com outras classes de investimento. Com os regimes capturando relativamente menos nuances da renda variável, o modelo se torna menos flexível, implicando em menor volume na alocação desta classe. No caso da renda fixa de longo prazo, como o modelo encontra rentabilidade superior na operação fortemente alavancada em renda fixa, a ponta comprada da operação é direcionada ao ativo correlacionado com melhor relação de risco e retorno. Assim, (i) embora o IMA-B 5+ apresente maior retorno esperado que o IMA-B 5; e (ii) porque há alta correlação entre ambos em todos os regimes (mas principalmente nos dois regimes mais comuns: $1 \mathrm{e}$ 2) - a menor volatilidade induz o maior volume da carteira ser direcionado à alocação em renda fixa de curto prazo.

Através da Tabela 2 é possível comparar as performances ano a ano e acumuladas no período completo entre 2009 e 2019. Como salientado, a estimação foi realizada totalmente fora da amostra para permitir a avaliação dos resultados de forma realista e passível de aplicações práticas.

Na Tabela 2 pode ser verificado um outro aspecto particular às características dos retornos em tela que leva à maior exposição à IMA-B5 e CDI em detrimento dos demais ativos. Tomando o ano de 2019 como exemplo, a Tabela 2 mostra que IBOV e IMA-B5+ foram os ativos mais rentáveis no ano, entretanto a carteira CGL apresenta retorno significativamente superior a esses ativos com baixa exposição a eles (como vimos na Figura 2). Ao mesmo tempo, as probabilidades históricas (dados da Figura 1) mostram que dentre as 52 semanas de 2019, o regime 2 vigorou como o mais provável em 47 delas. A Tabela 1 indica que sob o regime 2 as volatilidades de IBOV e IMAB5+ respectivamente seriam 10 e 5 vezes acima da volatilidade do IMA-B5. Enquanto o retorno esperado de ambos, respectivamente, seria "apenas" 5 e 3 vezes superior ao IMA-B5. Isso justifica a preferência do modelo a operar o IMA-B5 ao invés de IBOV e IMA-B5+ mesmo sob um cenário de alta rentabilidade esperada. Em outras palavras, além do fato de que ao trabalhar sob uma função dinâmica e não sob uma função estática como a função quadrática (média-variância), o modelo consegue realizar hedging demands - a alocação proposta pelo modelo CGL reflete o trade-off retorno versus volatilidade à exemplo de um investidor que analisa o índice de Sharpe e não apenas retornos em sua decisão.

\subsection{Performance fora da amostra}

$\mathrm{Na}$ análise ano-a-ano, a Tabela 2 mostra que os retornos do modelo CGL superam todos os benchmarks em 6 dos 10 anos observados na amostra. A magnitude dos retornos CGL, tanto para cima quanto para baixo, é visivel- 
Tabela 2

Performance comparativa em 10 anos de resultados

\begin{tabular}{|c|c|c|c|c|c|c|c|c|c|}
\hline & ano & CDI & IBOV & $\begin{array}{c}\text { PTAX + } \\
\text { T-Bill }\end{array}$ & $\begin{array}{c}\text { IMA-B } \\
5+\end{array}$ & $\begin{array}{c}\text { IMA-B } \\
5\end{array}$ & $\begin{array}{c}\text { modelo } \\
\text { CGL }\end{array}$ & $\begin{array}{c}\text { regime } \\
\text { único }\end{array}$ & $\begin{array}{l}\text { pesos } \\
\text { iguais }\end{array}$ \\
\hline 2019 & $\begin{array}{l}\text { retorno } \\
\text { vol } \\
\text { IS } \\
\text { turnover }\end{array}$ & $\begin{array}{l}6,1 \% \\
0,1 \%\end{array}$ & $\begin{array}{c}20,7 \% \\
17,7 \% \\
0,8\end{array}$ & $\begin{array}{c}10,3 \% \\
13,1 \% \\
0,3\end{array}$ & $\begin{array}{c}29,5 \% \\
8,7 \% \\
2,7\end{array}$ & $\begin{array}{c}13,3 \% \\
2,3 \% \\
3,2\end{array}$ & $\begin{array}{c}98,3 \% \\
34,6 \% \\
2,7 \\
18,6 \%\end{array}$ & $\begin{array}{c}29,5 \% \\
12,5 \% \\
1,9 \\
0,3 \%\end{array}$ & $\begin{array}{c}16,2 \% \\
4,5 \% \\
2,3 \\
0,5 \%\end{array}$ \\
\hline 2018 & $\begin{array}{l}\text { retorno } \\
\text { vol } \\
\text { IS } \\
\text { turnover }\end{array}$ & $\begin{array}{l}6,4 \% \\
0,1 \%\end{array}$ & $\begin{array}{c}22,8 \% \\
19,4 \% \\
0,8\end{array}$ & $\begin{array}{c}20,2 \% \\
13,1 \% \\
1,1\end{array}$ & $\begin{array}{c}13,5 \% \\
8,5 \% \\
0,8\end{array}$ & $\begin{array}{c}9,2 \% \\
2,8 \% \\
1,0\end{array}$ & $\begin{array}{c}-24,8 \% \\
37,0 \% \\
-0,8 \\
15,7 \%\end{array}$ & $\begin{array}{c}-2,3 \% \\
16,6 \% \\
-0,5 \\
0,3 \%\end{array}$ & $\begin{array}{c}14,8 \% \\
5,0 \% \\
1,7 \\
0,5 \%\end{array}$ \\
\hline 2017 & $\begin{array}{l}\text { retorno } \\
\text { vol } \\
\text { IS } \\
\text { turnover }\end{array}$ & $\begin{array}{l}10,5 \% \\
0,3 \%\end{array}$ & $\begin{array}{c}17,4 \% \\
18,9 \% \\
0,4\end{array}$ & $\begin{array}{c}-5,4 \% \\
10,2 \% \\
-1,6\end{array}$ & $\begin{array}{c}16,5 \% \\
9,4 \% \\
0,6\end{array}$ & $\begin{array}{c}13,2 \% \\
2,6 \% \\
1,0\end{array}$ & $\begin{array}{c}83,6 \% \\
44,6 \% \\
1,6 \\
17,4 \%\end{array}$ & $\begin{array}{c}33,3 \% \\
12,7 \% \\
1,8 \\
0,5 \%\end{array}$ & $\begin{array}{c}10,6 \% \\
4,5 \% \\
0,0 \\
0,5 \%\end{array}$ \\
\hline 2016 & $\begin{array}{l}\text { retorno } \\
\text { vol } \\
\text { IS } \\
\text { turnover }\end{array}$ & $\begin{array}{l}14,2 \% \\
0,2 \%\end{array}$ & $\begin{array}{c}32,1 \% \\
26,0 \% \\
0,7\end{array}$ & $\begin{array}{c}-9,9 \% \\
17,9 \% \\
-1,3\end{array}$ & $\begin{array}{c}25,6 \% \\
10,6 \% \\
1,1\end{array}$ & $\begin{array}{c}15,5 \% \\
2,7 \% \\
0,5\end{array}$ & $\begin{array}{c}-26,1 \% \\
53,5 \% \\
-0,8 \\
15,6 \%\end{array}$ & $\begin{array}{c}25,1 \% \\
18,3 \% \\
0,6 \\
0,5 \%\end{array}$ & $\begin{array}{c}15,7 \% \\
5,3 \% \\
0,3 \\
0,7 \%\end{array}$ \\
\hline 2015 & $\begin{array}{l}\text { retorno } \\
\text { vol } \\
\text { IS } \\
\text { turnover }\end{array}$ & $\begin{array}{l}12,9 \% \\
0,2 \%\end{array}$ & $\begin{array}{c}-14,9 \% \\
22,0 \% \\
-1,3\end{array}$ & $\begin{array}{c}50,1 \% \\
19,4 \% \\
1,9\end{array}$ & $\begin{array}{c}2,6 \% \\
11,9 \% \\
-0,9\end{array}$ & $\begin{array}{c}13,8 \% \\
3,0 \% \\
0,3\end{array}$ & $\begin{array}{c}-26,7 \% \\
41,1 \% \\
-1,0 \\
23,6 \%\end{array}$ & $\begin{array}{c}-5,4 \% \\
24,5 \% \\
-0,7 \\
0,8 \%\end{array}$ & $\begin{array}{c}12,0 \% \\
5,6 \% \\
-0,2 \\
0,7 \%\end{array}$ \\
\hline 2014 & $\begin{array}{l}\text { retorno } \\
\text { vol } \\
\text { IS } \\
\text { turnover }\end{array}$ & $\begin{array}{l}10,6 \% \\
0,1 \%\end{array}$ & $\begin{array}{c}6,2 \% \\
23,9 \% \\
-0,2\end{array}$ & $\begin{array}{c}8,9 \% \\
11,8 \% \\
-0,1\end{array}$ & $\begin{array}{c}21,0 \% \\
13,9 \% \\
0,7\end{array}$ & $\begin{array}{c}13,1 \% \\
2,6 \% \\
1,0\end{array}$ & $\begin{array}{c}70,0 \% \\
40,2 \% \\
1,5 \\
13,4 \%\end{array}$ & $\begin{array}{c}13,9 \% \\
20,5 \% \\
0,2 \\
0,5 \%\end{array}$ & $\begin{array}{c}12,6 \% \\
6,2 \% \\
0,3 \\
0,6 \%\end{array}$ \\
\hline 2013 & $\begin{array}{l}\text { retorno } \\
\text { vol } \\
\text { IS } \\
\text { turnover }\end{array}$ & $\begin{array}{l}7,8 \% \\
0,2 \%\end{array}$ & $\begin{array}{c}-8,3 \% \\
21,1 \% \\
-0,8\end{array}$ & $\begin{array}{c}10,3 \% \\
11,4 \% \\
0,2\end{array}$ & $\begin{array}{c}-15,0 \% \\
14,9 \% \\
-1,5\end{array}$ & $\begin{array}{l}3,2 \% \\
4,3 \% \\
-1,1\end{array}$ & $\begin{array}{c}-59,9 \% \\
54,9 \% \\
-1,2 \\
25,1 \%\end{array}$ & $\begin{array}{c}-18,5 \% \\
30,2 \% \\
-0,9 \\
0,7 \%\end{array}$ & $\begin{array}{c}-0,3 \% \\
6,0 \% \\
-1,3 \\
0,6 \%\end{array}$ \\
\hline 2012 & $\begin{array}{l}\text { retorno } \\
\text { vol } \\
\text { IS } \\
\text { turnover }\end{array}$ & $\begin{array}{l}8,7 \% \\
0,2 \%\end{array}$ & $\begin{array}{c}-0,6 \% \\
23,1 \% \\
-0,4\end{array}$ & $\begin{array}{c}15,5 \% \\
11,2 \% \\
0,6\end{array}$ & $\begin{array}{c}31,3 \% \\
8,8 \% \\
2,6\end{array}$ & $\begin{array}{c}15,2 \% \\
2,5 \% \\
2,5\end{array}$ & $\begin{array}{c}98,3 \% \\
33,9 \% \\
2,6 \\
21,1 \%\end{array}$ & $\begin{array}{c}33,5 \% \\
21,0 \% \\
1,2 \\
0,9 \%\end{array}$ & $\begin{array}{c}14,3 \% \\
4,3 \% \\
1,3 \\
0,5 \%\end{array}$ \\
\hline 2011 & $\begin{array}{l}\text { retorno } \\
\text { vol } \\
\text { IS } \\
\text { turnover }\end{array}$ & $\begin{array}{l}11,8 \% \\
0,2 \%\end{array}$ & $\begin{array}{c}-18,3 \% \\
21,3 \% \\
-1,4\end{array}$ & $\begin{array}{c}5,0 \% \\
12,7 \% \\
-0,5\end{array}$ & $\begin{array}{c}19,3 \% \\
6,5 \% \\
1,2\end{array}$ & $\begin{array}{c}17,1 \% \\
2,1 \% \\
2,5\end{array}$ & $\begin{array}{c}128,7 \% \\
30,0 \% \\
3,9 \\
17,6 \%\end{array}$ & $\begin{array}{c}46,2 \% \\
19,6 \% \\
1,8 \\
1,5 \%\end{array}$ & $\begin{array}{l}6,6 \% \\
4,3 \% \\
-1,2 \\
0,5 \%\end{array}$ \\
\hline 2010 & $\begin{array}{l}\text { retorno } \\
\text { vol } \\
\text { IS } \\
\text { turnover }\end{array}$ & $\begin{array}{l}9,5 \% \\
0,2 \%\end{array}$ & $\begin{array}{c}2,5 \% \\
20,8 \% \\
-0,3\end{array}$ & $\begin{array}{c}-0,1 \% \\
9,7 \% \\
-1,0\end{array}$ & $\begin{array}{c}19,3 \% \\
5,2 \% \\
1,9\end{array}$ & $\begin{array}{c}12,7 \% \\
1,2 \% \\
2,8\end{array}$ & $\begin{array}{c}54,7 \% \\
20,9 \% \\
2,2 \\
9,3 \%\end{array}$ & $\begin{array}{c}30,0 \% \\
10,6 \% \\
1,9 \\
0,8 \%\end{array}$ & $\begin{array}{l}9,1 \% \\
3,3 \% \\
-0,1 \\
0,4 \%\end{array}$ \\
\hline Acum & $\begin{array}{l}\text { retorno } \\
\text { vol } \\
\text { IS } \\
\text { turnover }\end{array}$ & $\begin{array}{l}9,8 \% \\
0,4 \%\end{array}$ & $\begin{array}{c}4,7 \% \\
21,5 \% \\
-0,2\end{array}$ & $\begin{array}{c}9,5 \% \\
13,4 \% \\
0,0\end{array}$ & $\begin{array}{c}15,5 \% \\
10,3 \% \\
0,6\end{array}$ & $\begin{array}{c}12,6 \% \\
2,7 \% \\
1,0\end{array}$ & $\begin{array}{c}21,6 \% \\
40,6 \% \\
0,3 \\
17,7 \%\end{array}$ & $\begin{array}{c}16,7 \% \\
19,5 \% \\
0,4 \\
0,7 \%\end{array}$ & $\begin{array}{c}11,1 \% \\
5,0 \% \\
0,2 \\
0,5 \%\end{array}$ \\
\hline
\end{tabular}

Nota: A tabela apresenta as performances das classes de ativos, da carteira CGL e de duas carteiras de referência para comparação. Os períodos anuais compreendem os retornos entre as estimações realizadas no início de cada dezembro. Já o período acumulado (Acum.) corresponde aos retornos obtidos entre as estimações de dezembro 2009 e dezembro 2019, e está apresentado anualizado. Os dados estão apresentados em retornos absolutos (e não em prêmio de risco). Retorno indica os retornos totais; vol, a volatilidade ao ano (desvio padrão anualizado); IS, o índice de Sharpe em base anual; enquanto turnover denota a rotação média da carteira por semana. 
mente maior que de seus pares, e isto decorre de uma alavancagem elevada - o que também se traduz por significativa volatilidade. Note-se que o índice Sharpe da carteira CGL foi superior às duas carteiras de referência em 5 dos 10 anos analisados, ao passo que venceu todas as classes de ativos (especialmente o IMA-B 5) em apenas 4 anos, o que se explica pela baixíssima volatilidade apresentada pelo IMA-B 5.

A tabela indica que os resultados do modelo CGL são obtidos a partir de um turnover médio de $18 \%$ por semana (girando historicamente entre $9 \%$ e 25\%) sobre o volume da carteira alavancada, bastante acima das carteiras pares - isso se dá porque a carteira CGL procura antecipar a mudança de regimes, que pode acarretar mudanças bruscas na carteira. Acompanhamos Chen et al. (2013) para determinar o cálculo do percentual de turnover como sendo o valor do montante negociado sobre o valor total da carteira, considerando, claro, a alavancagem. Segundo os autores, o valor do montante negociado pode ser obtido seja através do valor dos novos ativos comprados, seja através do valor absoluto dos ativos vendidos - uma vez que o consumo é desconsiderado. Entretanto, frisamos, que o montante negociado não corresponde à compra mais a venda semanal.

Os resultados acumulados durante toda a janela de estimações são apresentados na última parte da Tabela 2. Analisando os retornos obtidos, verificamos que entre os ativos destacam-se o IMA-B 5+ (15,5\% a.a.) e IMA-B 5 (12,6\% a.a.). Enquanto isso, a performance do modelo CGL supera-os, bem como supera as carteiras regime único (16,7\% a.a.) e pesos iguais $(11,1 \%$ a.a.), rendendo $21,6 \%$ ao ano no período! Esse resultado sugere que não apenas que a observância dos regimes é relevante na formação da carteira, mas também que o modelo se destaca da carteira ingênua $1 / n$, ratificando a importância do modelo CGL para estratégias que utilizam a gestão ativa do portfólio.

Para alcançar tal magnitude de retorno, o modelo CGL opera deveras alavancado. Utilizando o índice de Sharpe como métrica de retorno vis-a-vis o risco tomado, a volatilidade superior do modelo CGL é destacada através do índice de Sharpe menor do que nas classes de renda fixa e ligeiramente menor do que na carteia míope. Por outro lado, apesar de sua volatilidade, o modelo CGL supera os demais ativos e carteiras neste indicador de performance.

Para avaliar a robustez dos retornos do modelo CGL, observamos as séries semanais no período completo de análise (2010 a 2019). Realizamos dois testes de hipótese com a finalidade de avaliar se a média dos retornos da carteira CGL excederia a média dos retornos de cada um dos benchmarks (classes de ativos e carteiras de referência) com significância estatística.

Primeiro realizamos o teste não paramétrico Wilcoxon Rank Sum (tam- 
Tabela 3

P-valores da comparação de retornos médios entre o modelo CGL e os benchmarks

\begin{tabular}{lccrrrrr}
\hline teste & CDI & IBOV & $\begin{array}{r}\text { PTAX + } \\
\text { T-Bill }\end{array}$ & IMA-B 5+ & IMA-B 5 & $\begin{array}{r}\text { regime } \\
\text { único }\end{array}$ & pesos iguais \\
\hline Wilcoxon & 0,0001 & 0,0013 & 0,0002 & 0,0011 & 0,0002 & 0,0218 & 0,0001 \\
bootstrap & 0,0855 & 0,0819 & 0,1138 & 0,0624 & 0,0772 & 0,1203 & 0,0859 \\
\hline
\end{tabular}

Nota: A tabela apresenta os p-valores dos testes estatísticos, ambos realizados sob a hipótese nula de que a média dos retornos da carteira CGL não seria maior que a média dos benchmarks. O período testado corresponde aos retornos semanais entre 2 de dezembro 2009 e 4 de dezembro 2019. Wilcoxon corresponde ao teste unicaudal Wilcoxon Rank Sum and Signed Rank gerado a partir da função wilcox.test residente no programa estatístico R (R Core Team, 2019). Tal função acompanha os trabalhos de Hollander e Wolfe (1999) e Bauer (1972). Bootstrap refere-se ao teste de Efron e Tibshirani (1993). O resultado do bootstrap foi calculado com 1 milhão de amostras.

bém conhecido como teste Mann-Whitney) acompanhando Copeland e Friedman (1991) e Bergmann et al. (2018). A Tabela 3 apresenta os p-valores para a hipótese nula de que a média dos retornos da carteira CGL não seria maior que dos benchmarks. Note-se que todos os resultados ficaram abaixo de $5 \%$, indicando que no primeiro teste de robustez, a carteira CGL apresentou retornos acima dos benchmarks com significância estatística.

Reiczigel et al. (2005) afirmam que o teste Wilcoxon é amplamente utilizado para comparar séries com distribuição não-normal. Apesar disso, questionam a existência de premissas possivelmente falhas neste teste. Portanto, como sugerido pelos mesmos autores, adicionalmente apresentamos o teste de hipótese por bootstrap de Efron e Tibshirani (1993). A vantagem deste segundo teste é que gerando uma quantidade suficientemente elevada de novas amostras, ele permite o relaxamento da premissa quanto ao tipo de distribuição uma vez que sob a lei dos grandes números, é possível replicar as caraterísticas da distribuição original através do processo de reamostragem aleatória. A Tabela 3 também apresenta os p-valores deste teste, onde os resultados indicam significância estatística na ordem de $8 \%$, exceto na comparação com PTAX-T-Bill e regime único cuja significância foi aproximadamente $12 \%$.

Ainda que os resultados do segundo teste não tenham sido tão contundentes quanto no teste anterior, eles sugerem direção semelhante. Por isso, há necessidade de avaliação da robustez através de testes com premissas distintas. A análise conjunta fortalece a conclusão de que a performance da carteira CGL (em termos de retorno médio) supera estatisticamente as médias dos demais investimentos analisados.

As performances apresentadas nesta seção sugerem que a carteira CGL faz sentido e possua um nicho de mercado de interesse. Tal nicho engloba investidores com objetivos de longo prazo e que se sujeitem a volatilidades 
altas. Como a performance resulta de retornos significativamente alavancados, a estratégia levaria investidores de curto prazo - como, por exemplo, investimentos com horizonte de apenas um ano - a uma altíssima exposição de risco: para investidores com horizontes mais longos, tal exposição dissipa-se (ao menos, parcialmente). Ademais, a estratégia requer uma gestão bastante ativa (i.e., com rebalanceamentos semanais), indicando a necessidade de gestão profissional (i.e., com rebalanceamentos eficientes) e escala para operar sob custos transacionais controlados mesmo diante de elevado turnover.

\section{Conclusão}

Esta pesquisa inova na aplicação do modelo CGL com quatro classes de ativos de risco, e principalmente por ser a primeira avaliação da performance de uma alocação com estimações totalmente fora da amostra para um modelo de múltiplos regimes sob a função estocástica diferencial. Isto representa um passo importantíssimo neste campo da literatura internacional pois os resultados aqui obtidos são realistas e poderiam ser obtidos por gestores no mercado.

Mostramos que a performance média alcançada pelo modelo CGL superou todos os benchmarks (classes de ativos e carteiras de referência) em termos de retornos semanais médios acumulados entre dez/2009 e dez/2019, sempre com significância estatística. Nesse período, a nossa estratégia alcançou um retorno médio igual a $21,6 \%$ ao ano contra, por exemplo, $9,8 \%$ a.a. do CDI e 4,7\% a.a. do Ibovespa. E na análise separada de cada ano, em 6 dos 10 anos em tela, o modelo CGL apresentou média de retorno semanal superior a qualquer dos benchmarks. Tais performances corroboram os resultados anteriores que no entanto haviam sido obtidos dentro da amostra (Campani et al., 2020; Lewin e Campani, 2020).

Nossos resultados também indicam que ao superar estatisticamente os retornos médios da carteira igualmente ponderada, o modelo CGL se destaca na gestão ativa de portfólios. Da mesma forma, superando a carteira de regime único, torna-se evidente a importância da adoção do modelo de múltiplos regimes em estratégias de alocação de ativos.

Ainda assim, a estratégia proveniente do modelo CGL deve ser implementada por gestores profissionais, pois há necessidade de alto turnover semanal: os rebalanceamentos precisam ser feitos eficientemente e com custos transacionais controlados. Além disso, ela não é indicada para investidores de curto prazo, pois o risco pode ser alto demais para estes investidores: o dinamismo da estratégia ao trocar os regimes econômicos faz a mesma ter volatilidade acima dos índices tradicionais de mercado.

Os resultados encontrados nesta pesquisa motivam a extensão em novas aplicações do modelo CGL nos mercados brasileiro e internacional. Como 
próximos passos, avaliamos aplicar o modelo CGL separadamente à cada classe de ativo para permitir, por exemplo, ao investidor definir a própria calibragem entre renda fixa e renda variável durante o período de observação. Em outra frente, o fato de não incluirmos nos resultados custos de transação e de liquidez do mercado, aliados às elevadas posições vendidas, representam potenciais limitações à aplicação da estratégia e isto também deverá ser analisado em pesquisa futura. Não obstante, os resultados encontrados soam bastante promissores e abrem caminho para a utilização de modelos de múltiplos regimes na alocação de carteiras de investimento no Brasil.

\section{Agradecimentos}

Os autores agradecem ao apoio técnico e financeiro da Cátedra BRASILPREV em Previdência e ao apoio financeiro das seguintes instituições de fomento à pesquisa (em ordem alfabética): Associação Brasileira das Entidades dos Mercados Financeiro e de Capitais (ANBIMA); Conselho Nacional de Desenvolvimento Científico e Tecnológico (CNPq); Coordenação de Aperfeiçoamento de Pessoal de Nível Superior (CAPES); Escola de Negócios e Seguros (ENS); e Fundação Carlos Chagas Filho de Amparo à Pesquisa do Estado do Rio de Janeiro (FAPERJ).

\section{Referências}

Anbima (2020). Consolidado histórico de fundos de investimento - patrimônio líquido por classe Anbima. Acesso em: 28/05/2020.

URL: https: / /www.anbima.com.br/pt_br/informar/est atisticas/fundos-de-investimento/fi-consolidadohistorico.htm

Andrino, D. D. e Leal, R. P. C. (2018). Equally weighted strategic allocation and balanced funds in Brazil, Latin American Business Review 19(1): 2353.

URL: https://doi.org/10.1080/10978526.2018.1450150

Ang, A. e Bekaert, G. (2002). International asset allocation with regime shifts, Review of Financial Studies 15(4): 1137-1187.

URL: https ://doi .org/10.1093/rfs/15.4.1137

Ang, A. e Bekaert, G. (2004). How regimes affect asset allocation, Financial Analysts Journal 60(2): 86-99.

URL: https://doi.org/10.2469/faj.v60.n2.2612 
Ang, A. e Timmermann, A. (2012). Regime changes and financial markets, Annual Review of Financial Economics 4(1): 313-337.

URL: https://doi.org/10.1146/annurev-financial-11 0311-101808

Bae, G. I., Kim, W. C. e Mulvey, J. M. (2014). Dynamic asset allocation for varied financial markets under regime switching framework, European Journal of Operational Research 234(2): 450-468.

URL: https://doi.org/10.1016/j.ejor.2013.03.032

Bauer, D. (1972). Constructing confidence sets using rank statistics, Journal of the American Statistical Association 67(339): 687-690.

URL: https: //doi .org/10.1080/01621459.1972.104812 79

Bergmann, D., Savoia, J., De Angelo, C., Contani, E. e Silva, F. (2018). Portfolio management with tail dependence, Applied Economics 50(51): 5510 5520 .

URL: https://doi.org/10.1080/00036846.2018.1487000

Campani, C. H. e Garcia, R. (2019). Approximate analytical solutions for consumption/investment problems under recursive utility and finite horizon, North American Journal of Economics and Finance 48: 364-384.

URL: https://doi.org/10.1016/j.najef .2019.03.005

Campani, C. H., Garcia, R. e Lewin, M. (2020). Optimal portfolio strategies in the presence of regimes in asset returns, em revisão no Journal of Banking and Finance .

Case, B., Guidolin, M. e Yildirim, Y. (2014). Markov switching dynamics in REIT returns: Univariate and multivariate evidence on forecasting performance, Real Estate Economics 42(2): 279-342.

URL: https : //doi .org/10.1111/1540-6229.12025

Chen, F., Sanger, G. e Slovin, M. (2013). Asset sales in the mutual fund industry: Who gains?, Journal of Banking and Finance 37(12): 4834-4849.

URL: https://doi.org/10.1016/j.jbankfin.2013.08.0 19

Copeland, T. e Friedman, D. (1991). Partial Revelation of Information in Experimental Asset Markets, Journal of Finance 46(1): 265-295.

URL: https: //doi.org/10.1111/j.1540-6261.1991.tbo 3752.x 
DeMiguel, V., Garlappi, L. e Uppal, R. (2009). Optimal versus naive diversification: How inefficient is the $1 / \mathrm{N}$ portfolio strategy?, Review of Financial Studies 22(5): 1915-1953.

URL: https : //doi .org/10.1093/rfs/hhm075

Duffie, D. e Epstein, L. G. (1992). Stochastic Differential Utility, Econometrica 60(2): 353-394.

URL: https://www.jstor.org/stable/2951600

Efron, B. e Tibshirani, R. (1993). An Introduction to the Bootstrap, Chapman $\&$ Hall, New York.

Graflund, A. e Nilsson, B. (2003). Dynamic portfolio selection: The relevance of switching regimes and investment horizon, European Financial Management 9(2): 179-200.

URL: https://doi.org/10.1111/1468-036X.00215

Guidolin, M. (2011). Markov switching models in empirical finance, Advances in Econometrics 27(2): 1-86.

URL: https: //doi.org/10.1108/s0731-9053(2011) 00002 7B004

Guidolin, M. e Hyde, S. (2012). Can VAR models capture regime shifts in asset returns? A long-horizon strategic asset allocation perspective, Journal of Banking and Finance 36(3): 695-716.

URL: https://doi.org/10.1016/j.jbankfin.2011.10.0 11

Guidolin, M. e Ono, S. (2006). Are the dynamic linkages between the macroeconomy and asset prices time-varying?, Journal of Economics and Business 58(5-6): 480-518.

URL: https://doi.org/10.1016/j.jeconbus.2006.06.0 09

Guidolin, M. e Timmermann, A. (2007). Asset allocation under multivariate regime switching, Journal of Economic Dynamics and Control 31(11): 3503-3544.

URL: https://doi.org/10.1016/j.jedc.2006.12.004

Hamilton, J. D. (1989). A new approach to the economic analysis of nonstationary time series and the business cycle, Econometrica 57(2): 357-384.

URL: https : / /www. jstor.org/stable/1912559 
Hollander, M. e Wolfe, D. (1999). Nonparametric Statistical Methods, 2nd edn, John Wiley \& Sons, New York, www.jstor.org/stable/2063815.

Kessler, S. e Scherer, B. (2009). Varying risk premia in international bond markets, Journal of Banking and Finance 33(8): 1361-1375.

URL: https: //doi.org/10.1016/j.jbankfin.2009.02.0 07

Lewin, M. e Campani, C. H. (2020). Portfolio management under multiple regimes: Strategies that outperform the market, Revista de Administração Contemporânea 24(4): 300-3016.

URL: https : //doi .org/10.1590/1982-7849rac20201901 61

Munk, C. (2013). Financial Asset Pricing Theory, 6th edn, Oxford University Press, London.

Oliveira, A. B. e Pereira, P. L. (2018). Uncertainty times for portfolio selection at financial market. SSRN Working Paper no 473.

R Core Team (2019). R: A Language and Environment for Statistical Computing, R Foundation for Statistical Computing.

URL: https: / /www.r-project.org/

Reiczigel, J., Zakariás, I. e Rózsa, L. (2005). A bootstrap test of stochastic equality of two populations, American Statistician 59(2): 156-161.

URL: https : / /doi .org/10.1198/000313005X23526

Sangvinatsos, A. e Wachter, J. (2005). Does the failure of the expectations hypothesis matter for long-term investors?, Journal of Finance 60(1): 179230.

URL: https://doi.org/10.1111/j.1540-6261.2005.007 $28 . x$

Santiago, D. C. e Leal, R. P. C. (2015). Carteiras igualmente ponderadas com poucas ações e o pequeno investidor, Revista de Administração Contemporânea 19(5): 544-564.

Tu, J. (2010). Is regime switching in stock returns important in portfolio decisions?, Management Science 56(7): 1198-1215.

URL: https://doi.org/10.1287/mnsc.1100.1181 


\section{A. Apêndice}

\section{A.1 Detalhamento da configuração da economia}

Como os estados da economia não são observáveis, assumimos que o investidor enxerga os prêmios de risco como um processo de média ponderada pelas probabilidades de ocorrência de cada regime em $t$ :

$$
\mathrm{d} \hat{\boldsymbol{r}}_{t}=\boldsymbol{D}_{s, t} \boldsymbol{\pi}_{t} \mathrm{~d} t+\left(\boldsymbol{V} \boldsymbol{\pi}_{t}\right) \mathrm{d} \boldsymbol{Z}_{t},
$$

onde $\mathrm{d} \widehat{\boldsymbol{r}}_{t}$ é o vetor coluna com todos os $n$ processos dos prêmios de risco; $\boldsymbol{D}_{s, t}$ é o elemento configurado na equação (4) como a matriz $(n \times m)$ contendo os drifts dos ativos por regime; e $\boldsymbol{\pi}_{t}$ é um vetor coluna de dimensão é $m \times 1$ que arquiva as probabilidades (filtradas) de estar em cada possível estado da economia no momento $t$ condicionadas às informações disponíveis até aquele determinado momento $t$, sendo:

$$
\boldsymbol{\pi}_{\boldsymbol{t}}=\left[\begin{array}{llll}
\pi_{1, t} & \pi_{2, t} & \ldots & \pi_{m, t}
\end{array}\right]^{T},
$$

e $\boldsymbol{V}$ é um vetor linha de matrizes com dimensão $1 \times m$ que armazena as matrizes $\boldsymbol{\sigma}_{s, i}$ que, como detalhado na equação (3), são constantes e especificadas para cada regime $i \in R$, sendo $R=\{1,2, \ldots, m\}$ :

$$
\boldsymbol{V}=\left[\begin{array}{llll}
\boldsymbol{\sigma}_{s, 1} & \boldsymbol{\sigma}_{s, 2} & \ldots & \boldsymbol{\sigma}_{s, m}
\end{array}\right] .
$$

A propósito, Campani e Garcia (2019) testaram um modelo onde o investidor utiliza a média das matrizes de variância-covariância ao invés da média das matrizes de volatilidade, e afirmam que os resultados são virtualmente os mesmos.

As probabilidades de estado na equação (11) dadas por $\boldsymbol{\pi}_{t}$ são tratadas como variáveis de estado no modelo CGL e seu comportamento é determinado apenas pelos prêmios de risco observados. Assim, assumimos que tais probabilidades adotam o seguinte processo:

$$
\left[\begin{array}{c}
d \pi_{1, t} \\
d \pi_{2, t} \\
\ldots \\
d \pi_{m, t}
\end{array}\right]=\left[\begin{array}{c}
\sum_{i=1}^{m} \lambda_{i 1} \pi_{i, t} \\
\sum_{i=1}^{m} \lambda_{i 2} \pi_{i, t} \\
\ldots \\
\sum_{i=1}^{m} \lambda_{i m} \pi_{i, t}
\end{array}\right] \mathrm{d} t+\left[\begin{array}{cccc}
\sigma_{11, \pi} & \sigma_{12, \pi} & \ldots & \sigma_{1 n, \pi} \\
\sigma_{21, \pi} & \sigma_{22, \pi} & \ldots & \sigma_{2 n, \pi} \\
\ldots & \ldots & \ldots & \ldots \\
\sigma_{m 1, \pi} & \sigma_{m 2, \pi} & \ldots & \sigma_{m n, \pi}
\end{array}\right] \mathrm{d} \mathbf{Z}_{t},
$$

com as definições convenientes abaixo:

$$
\mathrm{d} \boldsymbol{\pi}_{\boldsymbol{t}}=\left[\begin{array}{c}
\mathrm{d} \pi_{1, t} \\
\mathrm{~d} \pi_{2, t} \\
\ldots \\
\mathrm{d} \pi_{m, t}
\end{array}\right] ; \boldsymbol{\mu}_{\boldsymbol{\pi}, t}=\left[\begin{array}{c}
\sum_{i=1}^{m} \lambda_{i 1} \pi_{i, t} \\
\sum_{i=1}^{m} \lambda_{i 2} \pi_{i, t} \\
\ldots \\
\sum_{i=1}^{m} \lambda_{i m} \pi_{i, t}
\end{array}\right] ; \boldsymbol{\sigma}_{\boldsymbol{\pi}}=\left[\begin{array}{cccc}
\sigma_{11, \pi} & \sigma_{12, \pi} & \ldots & \sigma_{1 n, \pi} \\
\sigma_{21, \pi} & \sigma_{22, \pi} & \ldots & \sigma_{2 n, \pi} \\
\ldots & \ldots & \ldots & \ldots \\
\sigma_{m 1, \pi} & \sigma_{m 2, \pi} & \ldots & \sigma_{m n, \pi}
\end{array}\right]
$$


onde $\mathrm{d} \boldsymbol{\pi}_{t}$ armazena os processos estocásticos seguidos pelas probabilidades $\pi_{i, t}$; $\boldsymbol{\mu}_{\boldsymbol{\pi}, t}$ armazena os drifts desses processos para cada regime e $\boldsymbol{\sigma}_{\boldsymbol{\pi}}$, as volatilidades parciais de cada um desses processos em relação a cada um dos elementos de Wiener que compõem o vetor $\mathrm{d} \boldsymbol{Z}_{t}$. Como definido na seção 3.2, os elementos do vetor $\mathrm{d} \boldsymbol{Z}_{t}=\left[\mathrm{d} Z_{1} \mathrm{~d} Z_{2} \ldots \mathrm{d} Z_{n}\right]$ são incrementos de processos de Wiener independentes entre si (sem nenhuma perda de generalidade). O processo de estimação da matriz $\boldsymbol{\sigma}_{\boldsymbol{\pi}}$ encontra-se detalhado no apêndice A.2.

\section{A.2 Detalhamento da estimação dos parâmetros do modelo}

Para esta aplicação do modelo CGL, assumimos que a periodicidade semanal seria suficientemente curta para razoavelmente igualarmos as probabilidades de transição de Hamilton (1989) com as densidades das probabilidades de transição de nossa aplicação. Ressaltamos que elas seriam idênticas caso tivéssemos considerado períodos infinitesimais $(\mathrm{d} t)$.

Com base nos processos apresentados na seção 3.2, temos:

$$
\operatorname{Pr}\left\{Y_{t+1}=j \mid Y_{t}=i\right\}=P_{i j, \Delta t=1}=P_{i j}=\frac{\lambda_{i j}}{-\lambda_{i i}}\left(1-e^{\lambda_{i i}}\right), \quad i \neq j,
$$

onde de forma conveniente escolhemos a unidade de tempo com a mesma frequência das informações (uma semana). Assim, obtemos as seguintes identidades:

$$
\lambda_{i i}=\ln P_{i i} \quad \text { e } \quad \lambda_{i j}=\frac{P_{i j} \ln P_{i i}}{1-P_{i i}} .
$$

Armazenaremos as probabilidades em tempo discreto (constantes) na matriz $P$ :

$$
\boldsymbol{P}=\left[\begin{array}{cccc}
P_{11} & P_{12} & \ldots & P_{1 m} \\
P_{21} & P_{22} & \ldots & P_{2 m} \\
\ldots & \ldots & \ldots & \ldots \\
P_{m 1} & P_{m 2} & \ldots & P_{m m}
\end{array}\right] .
$$

Conforme explicado por Hamilton (1989), assumimos que o investidor utiliza as probabilidades filtradas para inferir o regime do período atual e, assim, processar suas decisões ótimas de investimento. Esta inferência considera a iteração das seguintes equações:

$$
\widehat{\boldsymbol{Y}}_{t \mid t}=\frac{\widehat{\boldsymbol{Y}}_{t \mid t-1} \odot \boldsymbol{\eta}_{t}}{\mathbf{1}^{T}\left(\widehat{\boldsymbol{Y}}_{t \mid t-1} \odot \boldsymbol{\eta}_{t}\right)} \text { e } \quad \widehat{\boldsymbol{Y}}_{t+1 \mid t}=\boldsymbol{P}^{T} \widehat{\boldsymbol{Y}}_{t \mid t},
$$

onde 1 representa aqui um vetor de uns de dimensão $m \times 1$ e o símbolo $\odot$ denota uma multiplicação elemento-por-elemento. $\widehat{\boldsymbol{Y}}_{t \mid t}$ e $\widehat{\boldsymbol{Y}}_{t+1 \mid t}$, também de 
dimensões $m \times 1$, são vetores que contém as probabilidades filtradas de estar em cada regime, respectivamente nos momentos $t$ e $t+1$, dada a informação atualizada disponível no momento $t$. Finalmente, $\boldsymbol{\eta}_{t}$ é outro vetor $m \times 1$, cujos elementos são as densidades das probabilidades dos prêmios de risco no momento $t$ condicionadas por estar em cada regime. Para colocar em prática a iteração de $\widehat{\boldsymbol{Y}}_{t \mid t}$ e $\widehat{\boldsymbol{Y}}_{t+1 \mid t}$ necessitamos de um ponto de partida. Evidentemente que tal ponto pode ser considerado pelo investidor com base em suas expectativas sobre os estados da economia. Neste estudo, consideramos que o ponto de partida serão as probabilidades de longo prazo, também chamadas de probabilidades ergódigas ou probabilidades incondicionais, definidas por:

$$
\widehat{\boldsymbol{Y}}_{1 \mid 0}=\left(\boldsymbol{A}^{T} \boldsymbol{A}\right)^{-1} \boldsymbol{A}^{T} \boldsymbol{e}_{m},
$$

onde $\boldsymbol{e}_{m}$ denota o último vetor coluna da matriz identidade de ordem $m \times 1 \mathrm{e}$ $\boldsymbol{A}$ é uma matriz $m \times m$ onde as linhas correspondem às linhas de $\boldsymbol{I}_{m}-\boldsymbol{P}^{T}\left(\boldsymbol{I}_{m}\right.$ refere-se à matriz identidade de ordem $m$ ) e a última linha possui apenas $1^{\prime} s$. A demonstração desta fórmula consta em Hamilton (1989). Assumindo que estamos configurando um modelo de múltiplos regimes de tempo discreto, para encontrar o vetor $\boldsymbol{\eta}_{t, i}$, recordamos o processo seguido pelos ativos de risco, onde admite-se a seguinte solução:

$$
\left[\begin{array}{c}
\hat{r}_{1, t} \\
\hat{r}_{2, t} \\
\ldots \\
\hat{r}_{n, t}
\end{array}\right]=\left[\begin{array}{c}
a_{1, i}-\frac{1}{2} \sum_{k=1}^{n} \sigma_{1 k, i}^{2} \\
a_{2, i}-\frac{1}{2} \sum_{k=1}^{n} \sigma_{2 k, i}^{2} \\
\ldots \\
a_{n, i}-\frac{1}{2} \sum_{k=1}^{n} \sigma_{n k, i}^{2}
\end{array}\right] \mathrm{d} t+\left[\begin{array}{cccc}
\sigma_{11, i} & 0 & \ldots & 0 \\
\sigma_{21, i} & \sigma_{21, i} & \ldots & 0 \\
\ldots & \ldots & \ldots & \ldots \\
\sigma_{n 1, i} & \sigma_{n 2, i} & \ldots & \sigma_{n n, i}
\end{array}\right]\left[\begin{array}{c}
d Z_{1, t} \\
d Z_{2, t} \\
\ldots \\
d Z_{n, t}
\end{array}\right],
$$

tal que o elemento do vetor $\boldsymbol{\eta}_{t, i}$ ocupando a linha $i$ será:

$$
\boldsymbol{\eta}_{t, i}=\frac{1}{(2 \pi)^{\frac{n+1}{2}}\left|\boldsymbol{\sigma}_{i} \boldsymbol{\sigma}_{i}^{T}\right|^{\frac{1}{2}}} \exp \left[-\frac{1}{2}\left(\boldsymbol{L} \boldsymbol{R}_{t}-\boldsymbol{M L} \boldsymbol{R}_{i}\right)^{T}\left(\boldsymbol{\sigma}_{i} \boldsymbol{\sigma}_{i}^{T}\right)^{-1}\left(\boldsymbol{L} \boldsymbol{R}_{t}-\boldsymbol{M L} \boldsymbol{L} \boldsymbol{R}_{i}\right)\right],
$$

onde $\mathrm{LR}_{t}$ é um vetor $n \times 1$ de $n$ log-retornos dos prêmios de risco observados no momento $t$ e $\mathrm{MLR}_{i}$ armazena os log-retornos esperados condicionados por regime:

$$
\boldsymbol{L R}_{t}=\left[\begin{array}{c}
\hat{r}_{1, t} \\
r_{2, t} \\
\ldots \\
\hat{r}_{n, t}
\end{array}\right] \text { e } \boldsymbol{M} \boldsymbol{L} \boldsymbol{R}_{i}=\left[\begin{array}{c}
a_{1, i}-\frac{1}{2} \sum_{k=1}^{n} \sigma_{1 k, i}^{2} \\
a_{2, i}-\frac{1}{2} \sum_{k=1}^{n} \sigma_{2 k, i}^{2} \\
\ldots \\
a_{n, i}-\frac{1}{2} \sum_{k=1}^{n} \sigma_{n k, i}^{2}
\end{array}\right] .
$$

Os 48 parâmetros acima são estimados através da máxima verossimilhança (MLE) de acordo com o método apresentado por Hamilton (1989). 


\section{Tabela A1}

Valores estimados para a matriz $\sigma_{\pi}$

\begin{tabular}{lrrrr}
\hline & $Z_{1}$ & $Z_{2}$ & $Z_{3}$ & $Z_{4}$ \\
\hline regime 1 & $-3,61 \%$ & $2,90 \%$ & $-0,48 \%$ & $-0,96 \%$ \\
regime 2 & $2,95 \%$ & $-2,33 \%$ & $1,22 \%$ & $0,51 \%$ \\
regime 3 & $0,65 \%$ & $-0,56 \%$ & $-0,74 \%$ & $0,45 \%$ \\
\hline
\end{tabular}

Nota: A tabela apresenta a matriz $\boldsymbol{\sigma}_{\boldsymbol{\pi}}$ referente às volatilidades das probabilidades do modelo CGL com 3 regimes e 4 ativos de risco. Os valores correspondem aos dados da última janela de estimação fora da amostra, finda em 04.12.2019.

Os últimos parâmetros a serem definidos, de forma independente do procedimento anterior, são os elementos da matriz $\boldsymbol{\sigma}_{\boldsymbol{\pi}}$, introduzida pelo modelo CGL e definida acima na equação (13b). Para calculá-la, utilizamos $\boldsymbol{D}_{s, t}$ conforme definido na equação (4). Então calculamos os drifts semanais $\boldsymbol{D}_{s, t} \boldsymbol{\pi}_{t} \mathrm{e}$ a matriz de volatilidades $\boldsymbol{V} \boldsymbol{\pi}_{t}$, estando os termos $\boldsymbol{\pi}_{t}$ e $\boldsymbol{V}$ respectivamente definidos nas equações (11) e (12). Assim, a partir da equação (10), estimamos o processo discreto abaixo:

$$
\Delta \boldsymbol{Z}_{t}=\boldsymbol{V} \boldsymbol{\pi}_{t}^{-1}\left(\boldsymbol{L} \boldsymbol{R}_{t}-\boldsymbol{D}_{s, t} \boldsymbol{\pi}_{t}\right)
$$

onde $\boldsymbol{L} \boldsymbol{R}_{t}$ é o mesmo vetor coluna apresentado acima. Utilizamos as equações (13a) e (13b) para escrever a discretização $\left(\Delta \boldsymbol{\pi}_{t}-\boldsymbol{\mu}_{\boldsymbol{\pi}, t}\right)=\boldsymbol{\sigma}_{\boldsymbol{\pi}} \Delta \boldsymbol{Z}_{t}$ e armazenar o lado esquerdo da série temporal em uma matriz $m \times T$ denotada por $\left(\Delta \boldsymbol{\pi}-\boldsymbol{\mu}_{\boldsymbol{\pi}}\right)$. Também, armazenamos os termos $\Delta \boldsymbol{Z}_{t}$ em uma matriz $\Delta \boldsymbol{Z} n \times T$ (onde $T$ corresponde à duração da série temporal). Assim, finalmente, obtemos a estimação desejada:

$$
\boldsymbol{\sigma}_{\boldsymbol{\pi}}=\left(\Delta \boldsymbol{\pi}-\boldsymbol{\mu}_{\boldsymbol{\pi}}\right) \Delta \boldsymbol{Z}^{T}\left(\Delta \boldsymbol{Z} \Delta \boldsymbol{Z}^{T}\right)^{-1}
$$

A Tabela A1 apresenta os valores da matriz $\boldsymbol{\sigma}_{\boldsymbol{\pi}}$ para o modelo CGL com 3 regimes e os seguintes ativos de risco: IBOV, PTAX-T-Bill, IMA-B5+ e IMA-B5. 